\section{A technique to carry a torch for}

In 1961, American inventor Thomas Reed wrote, "This type of apparatus should be useful in high temperature research and engineering," referring to his latest invention-the inductively coupled plasma (ICP) torch. He could probably not have predicted, however, the vast influence this publication would have. Two decades later, the connection of an ICP torch to a mass spectrometer would transform elemental and isotope analysis techniques and add unprecedented analytical capabilities to the fields of chemistry, geology, archaeology, forensics and biology.

In ICP mass spectrometry (ICP-MS), an inductively coupled argon plasma torch is used to atomize and ionize a sample, which is usually introduced as an aerosol or by means of laser ablation. The singly charged ions are then transferred to a mass spectrometer through an orifice and analyzed, one charge-to-mass ratio at a time. Modern ICP-MS systems are rapid, allowing for trace measurements of multiple elements simultaneously. The technology can detect all metals and even some metalloids and non-metals, and it is highly sensitive: detection limits fall in the part-per-billion to part-pertrillion range, depending on the element being analyzed.

The process required to achieve such capabilities was long, set into motion by Reed's plasma-torch design. Most prior plasma torches had required the immersion of electrodes into gas, which often led to electrode disintegration. But Reed was able to create an electrode-free, stable plasma at atmospheric pressures by inductively heating the gas with a radiofrequency coil. It soon became clear that Reed's torch was extremely useful. In the mid-1960s, Stanley Greenfield and colleagues and Richard Wendt and colleagues reported that the ICP torch could be coupled to an atomic-emission spectrometer to detect trace metal ions in solution.

In the late 1970s, Alan Gray started to exper-

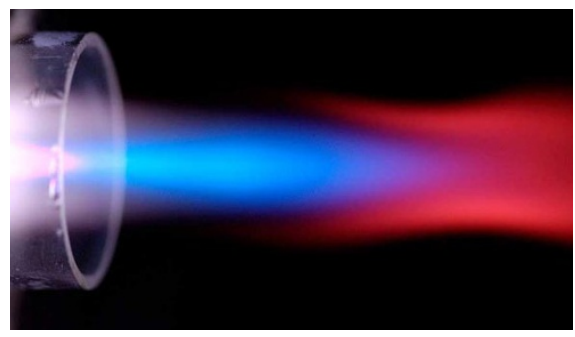

A plasma torch. Adapted from Aeschliman, D.B. et al., J. Anal At. Spectrom. 18, 1008-1014 (2003), with permission of the Royal Society of Chemistry.

iment with capillary-arc plasmas as potential ionization sources for mass spectrometry. Although his instrument had excellent detection limits, matrix effects and interference from ion species with similar mass-to-charge ratios were severe. The real breakthrough came in 1980, when Gray, together with researchers from the Ames Laboratory in lowa, decided to instead use an ICP torch, which could cleanly atomize and ionize the analyte to produce singly charged species.

The potential for elemental and isotope analysis was obvious immediately, not only to researchers but also to scientific-instrument manufacturing companies-most notably SCIEX and VG isotopes-which started to make the improvements that were needed for the technique to mature. For example, the extraction of the ICP ions, produced at atmospheric pressures, from the plasma into the vacuum system of the mass spectrometer required careful control of electrostatic effects. By 1988, the technology had advanced dramatically, and SCIEX and VG Isotopes had installed around 175 of these bulky instruments in laboratories around the world.

Modern ICP mass spectrometers have become substantially more compact, and there are numerous instruments currently in use worldwide. In geology labs, they are used to take reliable measurements of isotope ratios to determine the age and origin of liquid or solid samples. This application goes back to a development by VG Isotopes and Alex Halliday in the early 1990s known as multiple-collector ICP-MS, a type of mass spectrometry that separates ions into mass-resolved beams that are then guided to different collectors. With this technique, isotope ratios can be determined with a precision rate of $0.01-0.001 \%$. In 2009, Dmitry Bandura and colleagues reported the use of ICP-MS to simultaneously measure multiple antigens on single cells. To carry out this mass-cytometry technique, they attached lanthanide tags to antibodies against cell-surface antigens.

For Thomas Reed-who, despite entering retirement, has not stopped inventing-it must be incredibly rewarding to see how many useful applications his torch has sparked.

Leonie Mueck, Senior Editor,

Nature

ORIGINAL RESEARCH PAPERS Reed, T.B. Induction-coupled plasma torch. J. Appl. Phys. 32, $821-824$ (1961) | Houk, R.S. et al. Inductively coupled argon plasma as an ion source for mass-spectrometric determination of trace elements. Anal. Chem. 52, 2283-2289 (1980)

FURTHER READING Greenfield, S., Jones, I.L. \& Berry, C.T. High-pressure plasmas as spectroscopic emission sources. Analyst 89, 713-720 (1964) | Wendt, R.H. \& Fassel, V.A. Induction-coupled plasma spectrometric excitation source. Anal. Chem. 37, 920-922 (1965) | Houk, R.S. \& Thompson, J.J. Inductively coupled plasma spectrometry. Mass Spectrom. Rev. 7, 425-461 (1988) | Walder, A.J. \& Freeman, P.A. Isotopic ratio measurement using a double focusing magnetic sector mass analyser with an inductively coupled plasma as an ion source. J. Anal. At. Spectrom. 7, 571-575 (1992) | Bandura, D.R. et al. Mass cytometry: technique for real time single cell multitarget immunoassay based on inductively coupled plasma time-offlight mass spectrometry. Anal. Chem. 81, 6813-6822 (2009) 